\title{
Competition with Primary Sensory Afferents Drives Remodeling of Corticospinal Axons in Mature Spinal Motor Circuits
}

\author{
다u-Qiu Jiang, ${ }^{1}$ Boubker Zaaimi, ${ }^{1}$ and ${ }^{-}$John H. Martin ${ }^{1,2}$ \\ ${ }^{1}$ Department of Physiology, Pharmacology, and Neuroscience, City University of New York School of Medicine, The City College of New York, New York, \\ New York 10031, and ²Graduate Center, City University of New York, New York, New York 10016
}

\begin{abstract}
Injury to the mature motor system drives significant spontaneous axonal sprouting instead of axon regeneration. Knowing the circuitlevel determinants of axonal sprouting is important for repairing motor circuits after injury to achieve functional rehabilitation. Competitive interactions are known to shape corticospinal tract axon outgrowth and withdrawal during development. Whether and how competition contributes to reorganization of mature spinal motor circuits is unclear. To study this question, we examined plastic changes in corticospinal axons in response to two complementary proprioceptive afferent manipulations: (1) enhancing proprioceptive afferents activity by electrical stimulation; or (2) diminishing their input by dorsal rootlet rhizotomy. Experiments were conducted in adult rats. Electrical stimulation produced proprioceptive afferent sprouting that was accompanied by significant corticospinal axon withdrawal and a decrease in corticospinal connections on cholinergic interneurons in the medial intermediate zone and $\mathrm{C}$ boutons on motoneurons. In contrast, dorsal rootlet rhizotomy led to a significant increase in corticospinal connections, including those on cholinergic interneurons; $\mathrm{C}$ bouton density increased correspondingly. Motor cortex-evoked muscle potentials showed parallel changes to those of corticospinal axons, suggesting that reciprocal corticospinal axon changes are functional. Using the two complementary models, we showed that competitive interactions between proprioceptive and corticospinal axons are an important determinant in the organization of mature corticospinal axons and spinal motor circuits. The activity- and synaptic space-dependent properties of the competition enables prediction of the remodeling of spared corticospinal connection and spinal motor circuits after injury and informs the target-specific control of corticospinal connections to promote functional recovery.
\end{abstract}

Key words: activity dependent plasticity; corticospinal tract; motor cortex; proprioceptive afferents; rat

Significance Statement

Neuroplasticity is limited in maturity, but it is promoted after injury. Axons of the major descending motor pathway for motor skills, the corticospinal tract (CST), sprout after brain or spinal cord injury. This contributes to spontaneous spinal motor circuit repair and partial motor recovery. Knowing the determinants that enhance this plasticity is critical for functional rehabilitation. Here we examine the remodeling of CST axons directed by sensory fibers. We found that the CST projection is regulated dynamically in maturity by the competitive, activity-dependent actions of sensory fibers. Knowledge of the properties of this competition enables prediction of the remodeling of CST connections and spinal circuits after injury and informs ways to engineer targetspecific control of CST connections to promote recovery.

\section{Introduction}

Recovery of function after motor system injury in maturity is limited by a lack of motor pathway axon regeneration and, in

Received Sept. 15, 2015; revised Nov. 11, 2015; accepted Nov. 18, 2015.

Author contributions: Y.-Q.J., B.Z., and J.H.M. designed research; Y.-Q.J. and B.Z. performed research; Y.-Q.J. analyzed data; Y.-Q.J. and J.H.M. wrote the paper.

This work was supported by National Institutes of Health Grant 2R01NS064004 (J.H.M.), Craig H. Neilsen Foundation Grant 261214 (J.H.M.), and New York State Department of Health Spinal Cord Injury Research Board Grant C030172 (J.H.M.). We thank Hiroki Kimura for technical assistance and Xiuli Wu for histology and immunohistochemistry. We also thank Thomas Jessell for comments on a previous version of this manuscript. consequence, muted repair of damaged circuits (Silver et al., 2015). In contrast, significant sprouting of spared axons occurs after brain and spinal cord injuries (Oudega and Perez, 2012). Little is known about the determinants of axon sprouting. Pro-

The authors declare no competing financial interests.

Correspondence should be addressed to Dr. John H. Martin, Department of Physiology, Pharmacology, and Neuroscience, City University of New York School of Medicine, City College of New York, 160 Convent Avenue, New York, NY 10031. E-mail: jmartin@ccny.cuny.edu.

D0I:10.1523/JNEUROSCI.3441-15.2016

Copyright $\odot 2016$ the authors $\quad 0270-6474 / 16 / 360193-11 \$ 15.00 / 0$ 
moting motor pathway sprouting to strengthen spared connections may be an effective alternative to axon regeneration in motor system repair. Motor pathway sprouting contributes to spontaneous partial recovery or compensation of motor function after injury (Blesch and Tuszynski, 2002). By understanding what directs sprouting, we may ultimately be able to harness it for therapeutic control.

The focus of this study is how sprouting of the corticospinal tract (CST) is directed by primary afferent fibers. The CST and afferent fibers are the two major inputs to spinal sensorimotor circuits, and both are crucial for skilled movements (Marsden et al., 1984; Drew et al., 2004). In intact developing animals, sprouting in the corticospinal system, similar to many other neuronal systems, is complemented by axon withdrawal in establishing spinal motor circuits (Donatelle, 1977; Curfs et al., 1994; Gibson and Clowry, 1999; Martin et al., 1999; Martin and Lee, 1999; Li and Martin, 2000; 2002; Friel and Martin, 2005; Cesa and Strata, 2009). After spinal cord injury or unilateral pyramidal tract lesion (PTX) in maturity, the CST and afferent fibers reorganize within the spinal cord in reciprocal ways. The lesioned CST looses connections, and spared CST axons and muscle afferent fibers sprout into denervated regions (Brus-Ramer et al., 2007; Tan et al., 2012).

Synaptic competition is an important developmental mechanism for neural circuit construction, and it is understood to depend on the limited availability of target-derived factors (Deppmann et al., 2008) and neural activity (Personius and BaliceGordon, 2002; Martin, 2005). However, it is not known whether competition shapes CST and afferent fiber connections in maturity, as suggested by the reciprocal changes after injury. In development, the loosing systems withdraw their axons (Friel and Martin, 2005), but previous studies in mature animals have used injury models, PTX, and dorsal root section (Brus-Ramer et al., 2007; Tan et al., 2012; Darian-Smith et al., 2013), in which the loosing system in the proposed competition is also the lesioned system. Lesion studies are informative but alone do not answer the question of competition in maturity.

To determine the role of competition between spinal input pathways in shaping the distribution and strength of their connections in maturity, we manipulated forelimb primary afferent fibers in opposite ways in adult rats. In one set of experiments, we selectively increased the activity of proprioceptive afferents (PAs) of a single forelimb muscle by electrical stimulation (ES). We show that ES produced PA fiber sprouting on interneurons and motoneurons and, in turn, substantial CST axon withdrawal, fewer CST connections, and weaker motor cortex-to-muscle throughput. Complementary experiments used complete section of $\mathrm{C} 4$ to $\mathrm{C} 8$ dorsal rootlets [dorsal rootlets rhizotomy (DRX)] to reduce $\mathrm{PA}$ input. Whereas the loosing system is injured in these experiments, we show that the results are the opposite of ES. The winner is the CST, which makes more connections on spinal interneurons and produces stronger motor cortex-to-muscle throughput. These manipulations are robust; they can alter CST connection by $\sim 50 \%$.

This is the first demonstration of synaptic competition regulating mature motor systems spinal connections. Our findings show that CST inputs to spinal motor circuits can be regulated dynamically in maturity by the competitive actions of afferent inputs. The properties of the competition between the two systems enables prediction of the remodeling of spared CST connection and spinal motor circuits after injury and new ways to develop target-specific control of CST connections to promote functional recovery.

\section{A Experimental design}

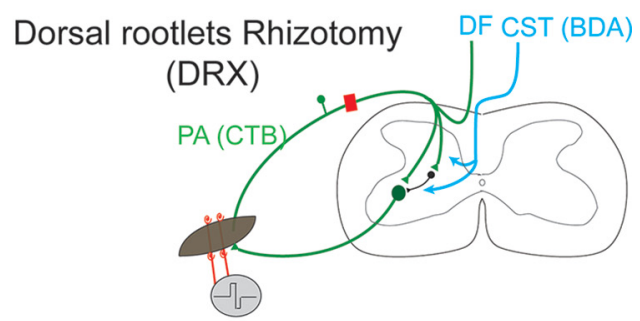

Electrical Stimulation (ES)

\section{B ES intensity control}

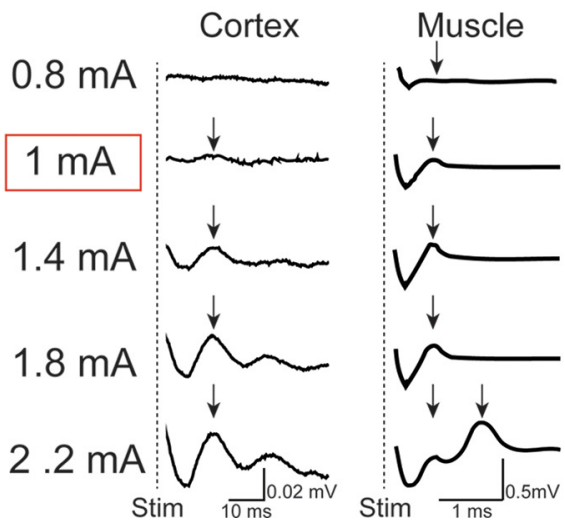

Figure 1. Experimental design. $\boldsymbol{A}$, Schematic illustration of the two manipulations to PAs: one is complete DRX and the other one is ES of ECR muscle PAs. $\boldsymbol{B}$, Recording for intensity control of ES in a representative animal. Simultaneous recording of SEPs (left) and ECR compound motor action potentials (right) evoked by muscle stimulation. Note that, starting from $1 \mathrm{~mA}$, the somatic SEP was observed at $\sim 10$ ms latency (arrows). The amplitude of the potential progressively increased with increasing stimulus amplitude. The muscle recording revealed an early response (single or left arrow) and, at $2.2 \mathrm{~mA}$, a later response. We interpret the initial muscle response as the $M$ wave and the second response the $H$ wave. In this representative case, we chose $0.9 \mathrm{~mA}$ ( $90 \%$ threshold for M wave and SEP) to chronically stimulate ECR.

\section{Materials and Methods}

Animals

Experiments were performed in accordance with the National Institutes of Health Guidelines for the Care and Use of Laboratory Animals. All animal protocols were approved by the City College Institutional Animal Care and Use Committee. Adult male Sprague Dawley rats (250-350 g) were housed under a $12 \mathrm{~h}$ light/dark cycle in a pathogen-free area with water and food provided ad libitum. All surgeries were performed under general anesthesia ( $90 \mathrm{mg} / \mathrm{kg}$ ketamine, $10 \mathrm{mg} / \mathrm{kg}$ xylazine, i.p.) and aseptic condition.

\section{$D R X$}

Animals were fixed in a stereotaxic frame under general anesthesia. Using $\mathrm{T} 2$ as a landmark, the left $\mathrm{C} 3$ to $\mathrm{T} 1$ cervical spinal vertebrae were exposed by hemilaminectomy. The C4 to C8 rootlets were carefully sectioned using microscissors without any damage to spinal cord (for experimental setup, see Fig. 1A). DRX animals were able to move the deafferented limbs as soon as they recovered from anesthesia, but the deafferented paw and wrist remained plantarflexed (Saling et al., 1992).

\section{Chronic intramuscular and epidural electrodes implantation}

Rats were anesthetized, and their head was fixed in a stereotactic frame. One end of a pair of stainless steel electrodes (Cooner Wire) was deinsulated and fixed in the belly of extensor carpi radials (ECR) muscle, $0.5 \mathrm{~cm}$ apart. The other end was tunneled under the skin from the distal forelimb via the neck to a connector fixed to the skull. A pair of silver ball elec- 
trodes was implanted epidurally in the caudal forelimb area of the motor cortex ( 2 and $0 \mathrm{~mm}$ anterior and $3 \mathrm{~mm}$ lateral to bregma). All wires were assembled in a six-channel pedestal (Plastics One) for easy access of either stimulation or recording electrodes.

\section{Chronic muscle ES}

To activate muscle PAs predominantly, low-frequency stimulation $(0.2$ ms biphasic pulse, one pulse, $1 \mathrm{~Hz}$ ) was delivered to the ECR muscle through implanted muscle electrodes $6 \mathrm{~h} / \mathrm{d}$, for $10 \mathrm{~d}$. Stimulation intensity was determined by recording somatosensory potentials (SEPs) through implanted cortical epidural ball electrodes (Sakatani et al., 1990) and muscle compound potentials through a pair of percutaneous nickelchrome wire electrodes (deinsulated $1 \mathrm{~mm}$ from the tip) in response to single-pulse ECR muscle stimulation at $1 \mathrm{~Hz}$. Both the cortical and muscle potentials were recorded with a differential alternating current amplifier (model 1700; A-M Systems) and filtered (bandpass 10-10 kHz). As shown in a representative recording in Figure $1 B$, the threshold of the response evoked in the cortex (SEP) is usually lower than that of the initial short-latency muscle response (Tan et al., 2012). At higher currents, muscle stimulation evoked a second, long-latency, muscle response (Song et al., 2015). We interpret the first response to be the $\mathrm{M}$-wave and the second response the $\mathrm{H}$-wave. Ninety percent of SEP threshold was used to activate preferentially muscle PAs to the spinal cord with minimal direct effects in the cortex, muscle fibers, and motoneurons. The threshold for the SEP was retested every other day during the stimulation period, and the current was adjusted as needed to be at $90 \%$ of threshold. ES animals did not show any visible behavioral changes during the stimulation period or after.

\section{Electromyography recordings}

We recorded motor evoked potentials (MEPs) in the ECR and biceps from the side that received the DRX or ES using percutaneous nickelchrome wire electrodes (deinsulated $1 \mathrm{~mm}$ from the tip) in response to trains of three stimuli to the contralateral motor cortex (biphasic pulse delivered with constant-current isolated stimulator, $0.2 \mathrm{~ms}$ per phase, 0.5 $\mathrm{Hz}$ repetition rate, $3 \mathrm{~ms}$ interstimulus interval for trains, $n=30$ sweeps). Electromyography (EMG) threshold was determined when nearly half of the 30 trains of stimuli were able to evoke EMG signals with averaged peak-to-peak amplitude $<0.05 \mathrm{mV}$. Then, currents of 1.0, 1.2, 1.4, 1.6, 1.8 , and 2 times threshold were delivered at an interval of $>2 \mathrm{~s}$ for the recruitment curve. In the DRX group, the MEP recordings were performed before, at $14 \mathrm{~d}$ after the lesion. In the ES group, the recordings were performed before, at $10 \mathrm{~d}$, and at 1 month after the end of ES. EMG latency also was examined. Latency was calculated as the difference between the onset of the stimulation artifact and the onset of the EMG signal. The onset of an EMG response was selected as the first increase in EMG activity that was more than two times SDs of the prestimulus baseline EMG activity.

In an additional three DRX animals, we used single-shock cortical stimuli to determine the latency of the ECR evoked response. Two hundred single-pulse stimuli $(0.2 \mathrm{~ms}$ biphasic, $2 \mathrm{~Hz})$ were delivered through epidural ball electrodes with an intensity of 1.4 to 1.6 times the three pulse thresholds. Onset latency of individual EMG responses to single stimuli were calculated from each sweep and then were plotted to obtain the scattered and frequency distribution.

\section{Anterograde labeling of CST axons}

Seven injections of biotinylated dextran amine (10\% BDA, $300 \mathrm{nl} /$ injection site; Invitrogen) were made into the forelimb area of the motor cortex that was contralateral to side of DRX or ES. Injections were made $1.5 \mathrm{~mm}$ below the pia surface with $\sim 500 \mu \mathrm{m}$ interval. BDA tracing was performed $14 \mathrm{~d}$ before the animals were euthanized.

\section{Muscle afferents tracing}

Cholera toxin B subunit (1\% CTB, $10 \mu$ l; List Biologicals) was injected bilaterally into the ECR using a Hamilton syringe. CTB injection was performed $7 \mathrm{~d}$ before the animals were euthanized.

\section{Tissue preparation and staining}

Animals were anesthetized deeply and perfused with saline, followed by $4 \%$ paraformaldehyde. Frozen tissues were cut transversely at $40 \mu \mathrm{m}$ thickness using a sliding microtome. BDA was visualized with $1 \%$ avidin-biotin complex reagent (ABC kit; Vector Laboratories), followed by the chromogen diaminobenzidine (DAB, Sigma) or streptavidin-FITC conjugate. Primary antibodies used in immunofluorescence staining include the following: goat anti-CTB (1:1000; List Biologicals), mouse antivesicular glutamate transporter 1 (VGLUT1; 1:10; NeuroMab), mouse anti-parvalbumin (PV; 1:1000; Millipore), and goat anti-choline acetyltransferase (ChAT; 1:1000; Sigma). The corresponding secondary antibodies are as follows: donkey anti-goat secondary antibody conjugated to FITC or Cy3; and donkey anti-mouse secondary antibody conjugated to Cy3 or Cy5. Sections were visualized and imaged digitally using a Zeiss LSM 710 meta confocal microscope.

\section{Anatomical data acquisition and analysis}

Regional CST axon length analysis. CST axon length was estimated using the "Space Balls" probe in the Stereo Investigator stereology program (MBF Science) as described in detail previously (Carmel et al., 2010). We represented changes in axon length within the gray matter using density maps. The contour of the gray matter and intersections between the virtual spheres in the Space Balls program and the labeled axons in the spinal cord contralateral to injection were used to create a TIFF file with intersections represented by a single pixel. Individual sections were corrected for orientation and aligned with one another according to fiduciary marks before being exported and quantified using custom programs written in MATLAB (MathWorks). Output files of individual sections of the spinal gray matter with labeled interactions were divided into $52 \times 52 \mu \mathrm{m}$ square regions of interest (ROIs). For each ROI, we used the number of interactions to compute an associated axon length. Length is represented according to a color scale, from the lowest density (blue) to the highest (red) with the associated numbers indicating an estimate of true axon length. Areas without labeling are coded black. Topographic heat map and total axon length estimates were corrected for tracing efficacy using the average number of BDA-labeled axons in the dorsal column, which was estimated using the Optical Fractionator in Stereo Investigator.

VGLUT1 and C bouton density analysis. VGLUT1 or C bouton signal was captured at $40 \times$ in $z$ stack containing individual motoneurons using confocal microscopy. The $z$-stack images were transferred to Neurolucida, contours were drawn along CTB- or ChAT-labeled motoneuron somas at each plane along the $z$ stack, and dendrites were drawn using neuronal tracing. Then, VGLUT1 or C boutons that contact the soma or dendrites were marked on the corresponding plane. The tracing files were then analyzed in Neurolucida Explorer to obtain estimates of soma surface area, dendrite length, and average local bouton number. We calculated VGLUT1 and C bouton density around motoneuron soma and on proximal dendrites.

Statistics. Statistical analyses were performed using Prism 5 (GraphPad Software) and Excel (Microsoft). The differences between groups were performed using repeated-measures ANOVA. Unpaired Student's $t$ test was used to compare the anatomy data between sham and chronic rhizotomy groups. Two-way ANOVA was used for comparing EMG recruiting curves between different time points before and after dorsal rhizotomy or muscle ES.

\section{Results}

\section{PA terminals sprout with muscle stimulation}

To visualize PA axons and changes produced by DRX and ES, we used three approaches: (1) PV immunohistochemistry to mark proprioceptive axons in the dorsal columns (Arber et al., 2000); (2) transganglionic tracing of PA axons with CTB from ECR; and (3) immunohistochemical staining of VGLUT1 to visualize muscle PA terminals contacting ECR motoneurons (Alvarez et al., 2004; Tan et al., 2012).

In control conditions, $\mathrm{PV}$ signal is enriched in the cuneate fasciculus, but it is nearly absent in the gracile fasciculus (Niu et al., 2013; Fig. 2A). CTB-labeled axons form a narrow and dense band extending from middle lamina III to medial lamina VI in the dorsal horn (Fig. 2E). CTB poorly labels PA terminals on 
A Ctrl

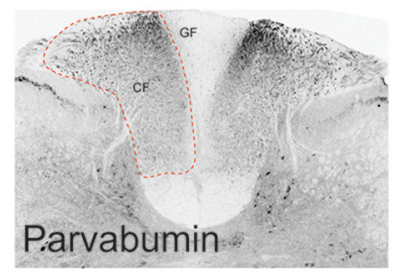

E Ctrl

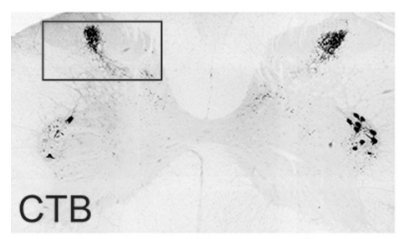

I Ctrl

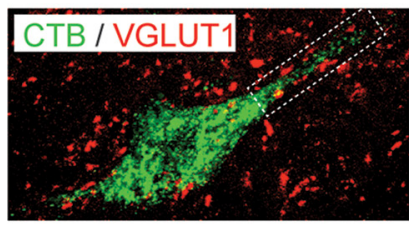

B ES

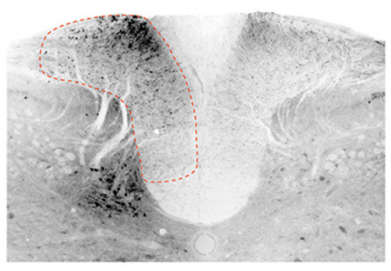

F ES

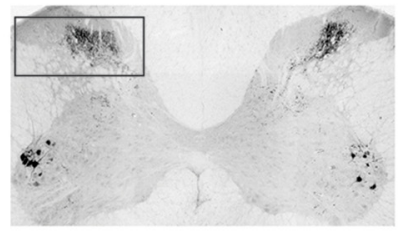

J ES

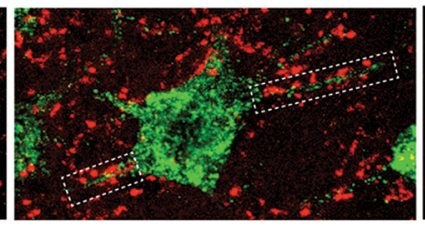

C DRX

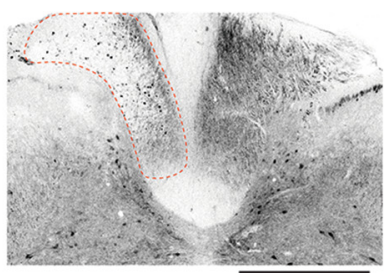

G DRX

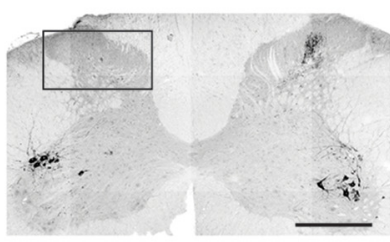

K DRX

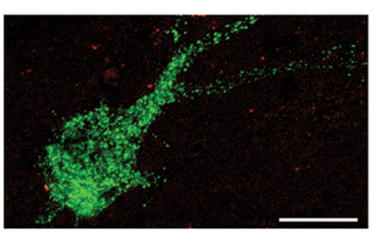

D PV density in DC

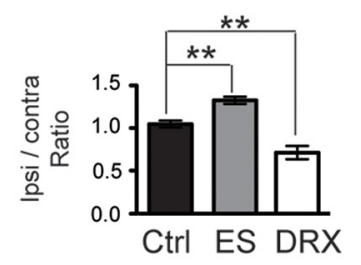

H Mediolateral plot

Normalized intensity Width at $50 \%$ peak
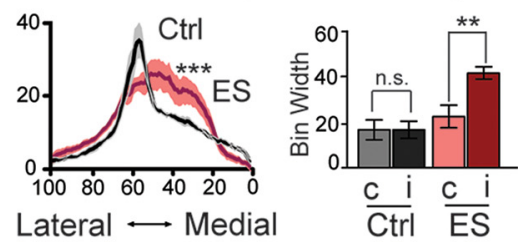

Figure 2. The activity and termination of PAs were modified by sensory manipulation. $A-C$, PV staining indicated activity of mechanosensory afferents in the cuneate fasciculus (CF). PV signal is much stronger right after $10 \mathrm{~d}$ of ES (B) but very weak 2 weeks after $\mathrm{DRX}$ ( $\boldsymbol{C}$. The ipsilateral/contralateral ratio (D) is significantly higher after ES and lower after DRX. $n=3$ for each group, one-way ANOVA with Dunnett's post hoc test; $p<0.01$ between control and DRX, $p<0.05$ between control and ES.E-G, CTB tracing of ECR PA termination in spinal dorsal horn. Note the territory expansion of CTB terminals right after $10 \mathrm{~d}$ of ES $(\boldsymbol{F})$ and absence of CTB 2 weeks after DRX (G). The mediolateral (ML) distribution of (TB signals in the dorsal horn $(\boldsymbol{H})$ is significantly different in the ES group compared with control (two-way ANOVA, ${ }^{* * *} p<0.001$ ). The width of the ML curve is significantly larger in ES on the ipsilateral side than that of ES on the contralateral side (paired $t$ test between ctrl-contra and ctrl-ipsi or between ES-contra and ES-ipsi, ${ }^{* *} p<0.01, n=3$ for control, $n=5$ for ES). I- $\boldsymbol{K}$, VGLUT1 labeling revealed proprioceptive projection on motoneurons. Note there are more VGLUT1 terminals on motoneuron soma and dendrites right after $10 \mathrm{~d}$ of ES $(\boldsymbol{J})$ and complete loss of VGLUT1 terminals 2 weeks after DRX (K). VGLUT1 bouton density analysis ( $\boldsymbol{L}$ ) showed that the increase of VGLUTA1 density on the ipsilateral side is significantly larger compared with the contralateral side on ES (paired $t$ test, ${ }^{*} p<0.05$ between ES contra and ES ipsi; $n=4$ for control, $n=$ 4 for ES). Scale bars: $A, B, 500 \mu \mathrm{m} ; C, 20 \mu \mathrm{m}$.

motoneurons, but VGLUT1 is effective. Large VGLUT1-positive $\left(\mathrm{VGLUT}^{+}{ }^{+}\right.$) boutons are enriched around the soma and proximal dendrites of ECR motoneurons, which are labeled retrogradely with CTB (Fig. 2I). Although CST axonal terminals also contain VGLUT1 (Fig. 3A; Valtschanoff et al., 1993; Du Beau et al., 2012), direct CS-motoneuron projections are found to be lacking or very rare in rodents (Yang and Lemon, 2003; Alstermark et al., 2004). Importantly, we measured the size of $\mathrm{CTB}^{+} /$ $\mathrm{VGLUT}^{+}$and $\mathrm{BDA}^{+} / \mathrm{VGLUT}^{+}$punta, respectively, and found that the size of $\mathrm{CTB}^{+} / \mathrm{VGLUT1}^{+}$puncta (mean, $3.998 \pm$ $\left.0.294 \mu \mathrm{m}^{2}\right)$ is significantly larger than that of BDA ${ }^{+} / \mathrm{VGLUT}^{+}$ puncta (mean, $0.6762 \pm 0.029 \mu \mathrm{m}^{2} ;{ }^{\star * *} p<0.001$, unpaired $t$ test; Fig. $3 B$ ). Therefore, we consider that these large VGLUT1 boutons surrounding motoneurons are very likely muscle PA terminals.

Immediately after $10 \mathrm{~d}$ of ES, we found significantly more PV labeling in the cuneate fasciculus on the stimulated side (Fig. $2 B, D ; p<0.05$, one-way ANOVA). Given that PV expression is highly correlated with neuronal activity in the visual and motor cortices (Cellerino et al., 1992), the elevated PV signal in ES animals shows that ECR ES was effective in increasing the activity of forelimb PAs. The stimulated PAs, instead of being confined to a narrow area as in controls (Fig. $2 E$ ), expanded their territory within the dorsal horn from the central to the more medial part of laminae II-III (Fig. 2F). The mediolateral distribution of PA terminals was broadened significantly in ES animals compared with controls (Fig. $2 \mathrm{H}$; ${ }^{* * *} p<0.001$, two-way ANOVA). Additionally, there was an $\sim 55 \%$ increase in VGLUT1 bouton density on motoneuronal soma and $\sim 44 \%$ increase on proximal dendrites (Fig. $2 \mathrm{~J}, L$ ). Together, these findings suggest that ECR muscle afferents not only sprout in the dorsal horn but also make more connections with motoneurons in response to the higher activity produced by chronic ES.

Two weeks after DRX, as expected, there is a significant reduction in PV labeling in the cuneate fasciculus (Fig. 2C,D; $p<0.01$, one-way ANOVA) and near complete loss of CTB signal in the dorsal horn on the lesion side (Fig. 2G). Residual CTB transganglionic label is explained by spared ECR afferent axons that enter the spinal cord caudal (or possibly rostral) to the denervated rootlets. Large VGLUT1 ${ }^{+}$boutons surrounding CTB-labeled motoneurons and in nearby motor neuron pools almost completely disappeared (Fig. $2 K$ ), confirming that the majority of large VGLUT1 ${ }^{+}$boutons are from afferent fibers. These findings confirmed elimination of the ECR proprioceptive projection in the spinal cord with DRX (Jiang et al., 2013). It thus provides a robust and simple complementary model to ES in terms of PA changes for studying the response of CST projections. 
A
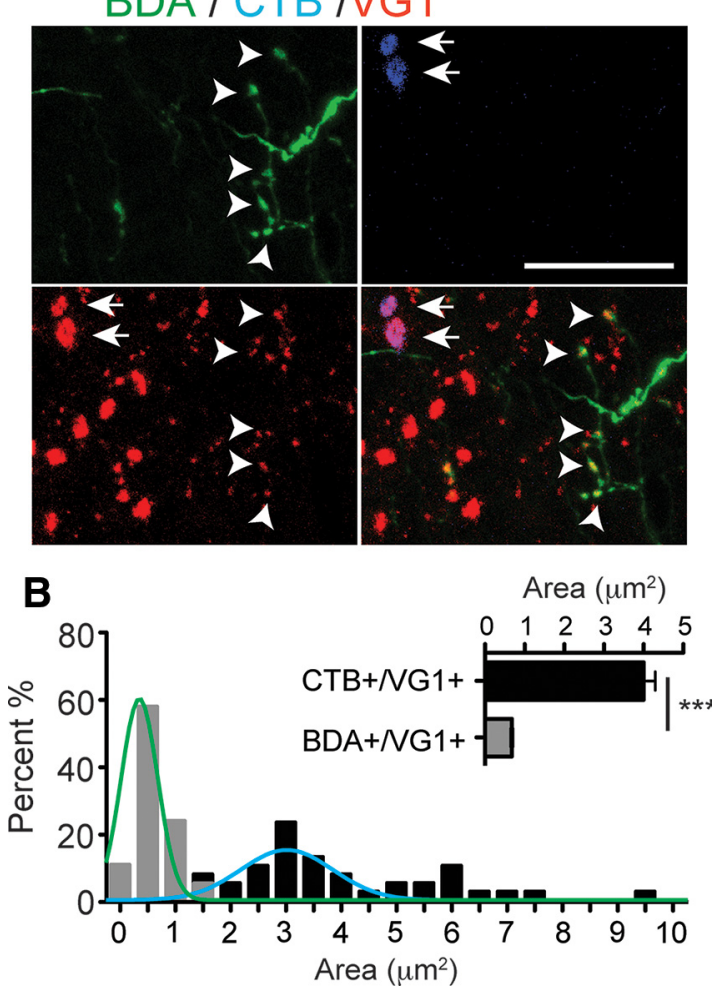

Figure 3. Proprioceptive and CS terminals colabel with different-sized VGLUT1 puncta. $\boldsymbol{A}$, Colabeling of (TB (arrows) or BDA (arrowheads) with VGLUT1 in the spinal cord. Scale bar, 20 $\mu \mathrm{m} . B$, Size distribution of BDA ${ }^{+} / \mathrm{NGLUT1}{ }^{+}$or $\mathrm{CTB}^{+} / \mathrm{NGLUT1}{ }^{+}$boutons. Each group fits well with Gaussians distribution. Inset histograms are averaged VGLUT1 bouton size in each group. ${ }^{* * *} p<0.001$, unpaired $t$ test.

\section{Afferent fiber manipulations reciprocally modulate the CST projection}

We next determined whether changes in afferent input-either activation of PAs by muscle ES or loss attributable to DRX - produced changes in CST connections. In control animals, averaged regional distributions of contralateral CST axons, corrected for tracing efficacy, show that the contralateral CST projects throughout most of the dorsal and intermediate spinal gray matter, but it is much denser in the medial dorsal horn and medial intermediate zone (IZ; Fig. 4Ai). Surprisingly, after ES, there was a much lower density of CST axons in the medial dorsal horn, normally the region with densest terminations, and a smaller high-density focus as well (Fig. 4Aii). This decrease in gray matter CST axon density occurred without any discernible change in the white matter axon density. Normalized total axon length in the ES group showed a substantial decrease $\left(\sim 40 \%\right.$; Fig. $4 B$; ${ }^{* *} p<$ 0.01 , one-way ANOVA), indicating the loss of CST axons. In comparison, after DRX, there was a similar topographic organization as control (Fig. 4Aiii). Normalized total CST axon length after DRX is also comparable with controls (Fig. 4B). Whereas enhancing PA activity strongly drives retraction of CS axons, deafferentation does not affect the general distribution of CST axons.

CST presynaptic sites in the middle IZ and the lateral motoneuron pool were identified by BDA and VGLUT1 colocalization (Fig. 4C,D). These two regions were chosen because they contain premotor interneurons or motoneurons (Stepien et al., 2010; Asante and Martin, 2013). Normally, there is an abundance of VGLUT1 ${ }^{+}$puncta distributed within these two areas (Fig. $4 \mathrm{Ci}, \mathrm{Di}$ ). CST boutons colocalize with small $\mathrm{VGLUT}^{+}{ }^{+}$puncta
(Fig. $4 C, D$, arrow), indicating that they are active excitatory presynaptic terminals formed by CST axons (Friel et al., 2012). We measured VGLUT1 ${ }^{+}$CST bouton density along BDA-labeled axons to determine the CST functional connection within these two areas. Interestingly, there was no difference in CST bouton density between ES and controls in both the IZ and lamina IX (Fig. 4Cii,Dii, F), which suggests that the remaining CS axons maintained their capacity of synaptic connection. Importantly, with the substantial loss of CST axon length after ES, the total number of CST connections onto spinal neurons in response to augmenting PA activity undergoes a substantial reduction.

After DRX, although the large VGLUT1 boutons of PAs are completely eliminated in the IZ and lamina IX, small VGLUT1 boutons are detected that colabeled with CST axon varicosities (Figs. 3A, 4Ciii,Diii). CST bouton (i.e., BDA + VGLUT1) density analysis revealed an $\sim 80 \%$ increase in the IZ and $76 \%$ increase in lamina IX compared with that of controls (Fig. 4F). This indicates that the loss of afferent fibers led to a significant increase in the density of excitatory CST connections but no overall expansion of the territory. Whereas we were unable to quantify the increase in CST contacts on motoneurons because they are too sparse to be measured reliably in the control and ES animals, we regularly observed direct contacts of VGLUT1 ${ }^{+}$CS boutons on the proximal dendrites of CTB-labeled ECR motoneurons in the lateral motor neurons pool after DRX (Fig. 4E). We propose that these newly formed contacts between the CST and motoneurons after DRX enable a more efficient throughput from motor cortex to muscle but now along a more direct pathway to the ventral horn.

\section{CST axons establish more projections on cholinergic partition interneurons in response to deafferentation}

We hypothesize that the reciprocal changes in CST axons and bouton density in the IZ after PA manipulations affect CST targeting of interneurons, as we have just shown for motoneurons. To examine this question further, we studied cholinergic interneurons in the medial IZ area, the so-called partition interneurons (Barber et al., 1984). These interneurons include a subset that is premotor (Miles et al., 2007; Asante and Martin, 2013). We examined CST axon contacts directly on $\mathrm{ChAT}^{+}$interneurons in the medial portion of lamina VII. We frequently detected CST axon contacts onto $\mathrm{ChAT}^{+}$interneurons within this area in controls (Fig. 5A). Interestingly, examination of relatively complete $z$ stacks of individual $\mathrm{ChAT}^{+}$interneurons revealed a trend whereby the farther from the dorsal column, the lower the likelihood that interneurons receive a direct CST projection (Fig. 5Ai-Aiii).

We randomly selected 10 sections from each of four animals in each group to determine the average number of CST boutons on cholinergic interneurons. After ES, we found an $\sim 45 \%$ decrease in CS bouton density (Fig. $5 B ;{ }^{\star} p<0.05$ between control and ES, one-way ANOVA with Dunnett's post hoc test). The cumulative distribution of bouton density (Fig. 5C) in ES animals shifted to the left of control, indicating that more $\mathrm{ChAT}^{+}$ interneurons have a lower density of CST terminations. The decreased CST bouton density suggests that cholinergic interneurons in ES animals have a diminished capacity to mediate CST actions on motoneurons. In contrast to ES, we found a $48 \%$ increase in average CS bouton density on cholinergic interneurons after DRX (Fig. $5 B ;{ }^{* *} p<0.01$ between control and DRX, one-way ANOVA with Dunnett's post hoc test). The cumulative distribution curve shifted to the right, which indicates that more $\mathrm{ChAT}^{+}$interneurons receive a higher density of CST termina- 
A i. Ctrl

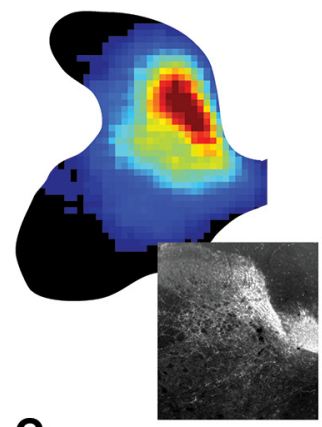

C BDAIVGLUT1

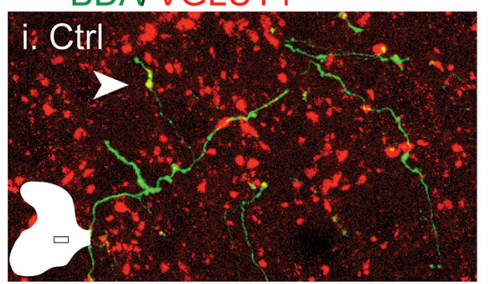

D

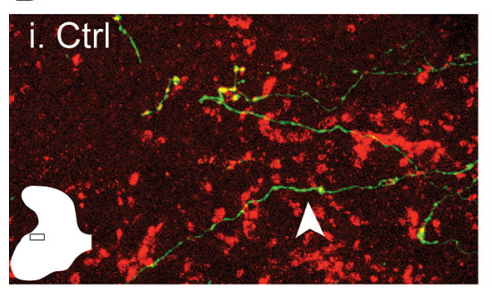

ii. ES

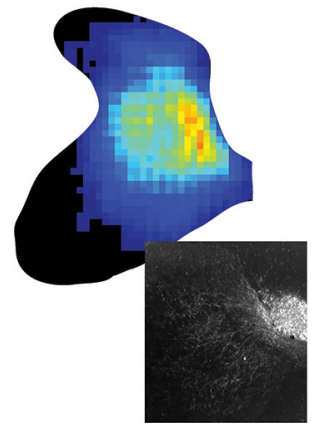

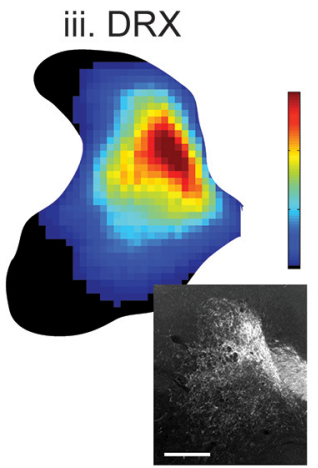

B

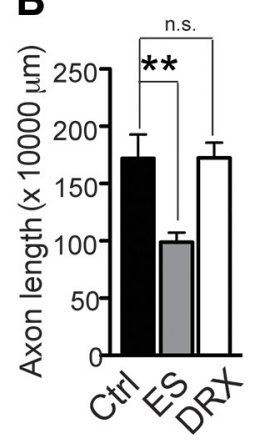

E

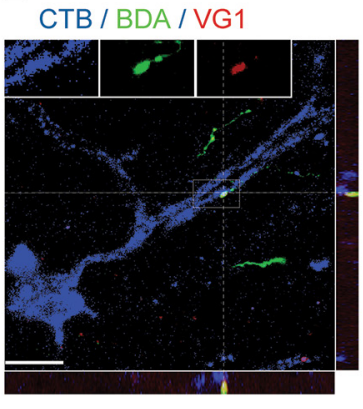

$\mathbf{F}$

Lam VII
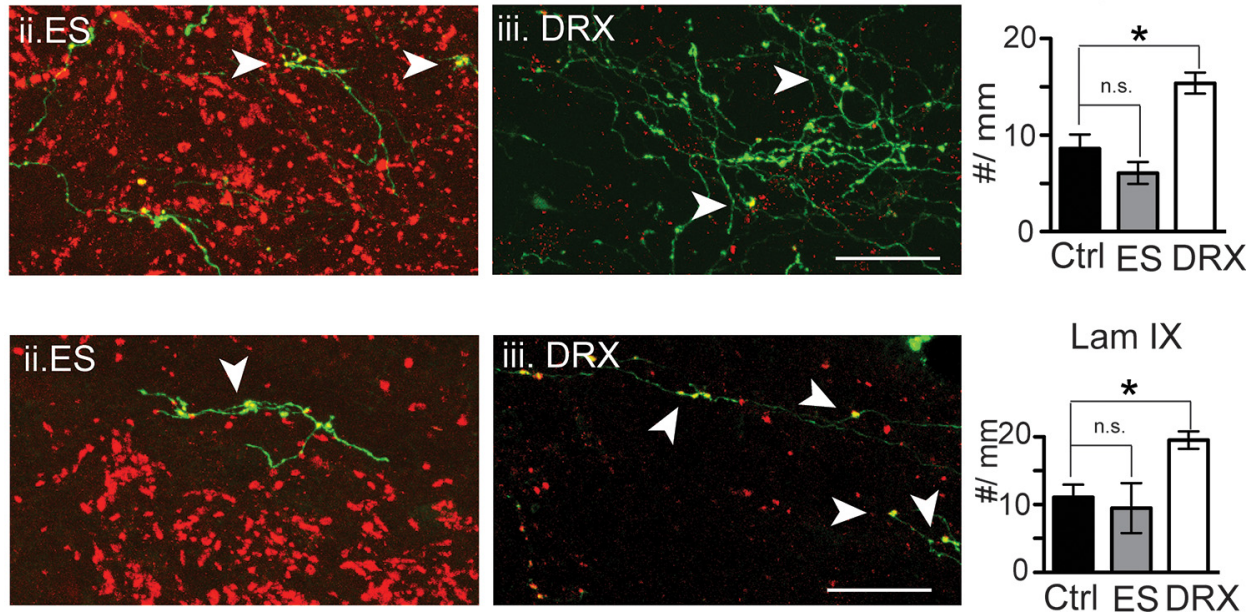

Figure 4. Corticospinal axons were reciprocally modified in response to PA manipulation. $A$, Axon density map. ES induces remarkable decrease of axon density in spinal gray matter (Aii) compared with control (Ai). DRX showed similar axon distribution pattern (Aiii). The bottom shows representative original DAB-staining images of BDA-traced CST axons in each group. The raw images were inverted to enable better visualization of the labeling. $B$, Estimated total axon length indicated significantly less axons in ES group (one-way ANOVA, $p>0.05$ between control and DRX, ${ }^{*} p<0.05$ between control and ES; $n=3$ for control, $n=5$ for DRX and ES). $\boldsymbol{C}, \boldsymbol{D}$, Presynaptic (S terminals are modified in both intermediate zone (C) and motor neuron pools (D). There is not much visible change in CS boutons in ES (Cii, Dii). VGLUT1 ${ }^{+}$puncta are almost absent after DRX, but there seem to be more VGLUT1 colabled CS boutons (Ciii, Diii) compared with control (Ci, Di). E, Direct CS projection on motoneuron dendrites was detected after DRX. Inset showed colocalization of BDA with VGLUT1. F, VGLUT1 ${ }^{+}$CS bouton density analysis. DRX induced significant increase in CS bouton density in both medial lamina VII (IZ) and motor neuron pool, and there is no change in ES animals (one-way ANOVA with Dunnett's multiple comparison post hoc test, ${ }^{*} p<0.05$ between control and DRX; $n=3$ for control, $n=5$ for DRX and ES). Scale bars: $\boldsymbol{A}, 0.5 \mathrm{~mm} ; \boldsymbol{C}-\boldsymbol{E}, 20 \mu \mathrm{m}$.

tions in DRX animals (Fig. 5C). With presumably more CST inputs conveyed by extra connections, these cholinergic interneurons potentially comprise a greater excitatory projection for the CST to motoneurons after deafferentation.

We next examined cholinergic interneuron terminals on motoneurons, the large $\mathrm{C}$ boutons. Using ChAT staining, we labeled both motoneurons and $\mathrm{C}$ boutons. Normally, $\mathrm{C}$ boutons preferentially contact the motoneuron soma than dendrites (Fig. 5Di). We compared $\mathrm{C}$ bouton density surrounding motoneuron somas on both sides within each groups. ES animals showed fewer C boutons contacting the motoneuron soma, but it is significantly higher in DRX animals (Fig. 5Dii,Diii,E; paired $t$ test, $p>0.05$ between the contralateral and ipsilateral sides in controls; ${ }^{*} p<$ 0.05 between the contralateral and ipsilateral sides in ES rats and ${ }^{*} p<0.01$ within DRX). We noticed that there are also more C boutons on the proximal dendrites of DRX motoneurons compared with controls (Fig. 5Diii), suggesting a more prominent cholinergic modulation of motoneurons excitability after DRX. $\mathrm{C}$ bouton density ratios between the contralateral to ipsilateral sides indicate an $\sim 15 \%$ decrease in ES animals and 58\% increase in DRX (Fig. $5 F$ ). The changes in $\mathrm{C}$ bouton density parallel changes in CST terminations on cholinergic interneurons in the medial IZ as either a decrease or increase. These findings suggest that the reciprocal changes in $\mathrm{C}$ bouton numbers with $\mathrm{ES}$ and DRX could lead to corresponding changes in motoneuron excitability.

\section{M1 motor-evoked muscle effects were modified correspondingly}

To determine whether changes in CST connections produced by afferent fiber manipulations influences signal transmission from the motor cortex to muscle, we recorded MEPs in ECR and biceps muscles in response to M1 stimulation. Ten days of ES produced a marked reduction in MEP amplitude in ECR, the stimulated muscle, and biceps (Fig. 6A). The reduction in MEP amplitude is well demonstrated by recruitment curves for the population of animals. Under naive conditions, response amplitude becomes larger with increasing M1 stimulation intensity between threshold and twice threshold. The recruitment relation was significantly decreased in both muscles (Fig. $6 B, C$ ) but much greater in ECR than biceps (Fig. 6D; $\sim 50 \%$ decrease in ECR vs $\sim 20 \%$ in biceps). Interestingly, the recruitment curve at 4 weeks after cessation of ES is not significantly different from the naive animals (Fig. 6A-C), showing that CST withdrawal and associated decreased connection strength is not permanent. 
A

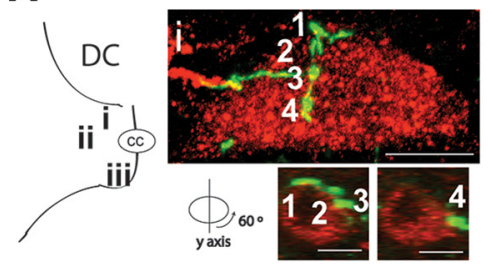

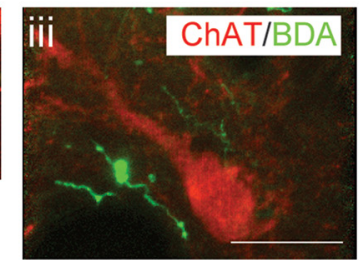

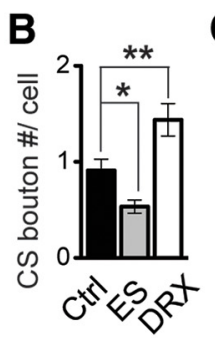

E

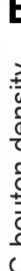

C

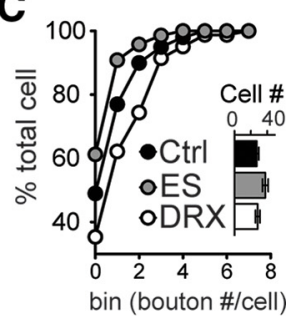

$\mathbf{F}$
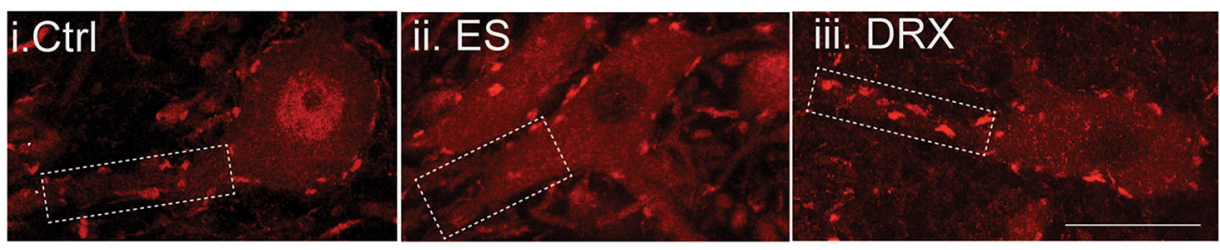

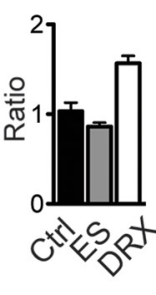

Figure 5. Cholinergic interneuron input and output properties were modified responsively. $A$, Corticospinal axons directly project to cholinergic interneurons in medial IZ. $z$ stack projection images of three cholinergic interneurons at different locations in the medial IZ area (as indicated in the schematic figure on left) were shown as examples. Insets are single-plane images showing the direct contact between CS boutons and ChAT neuron in $\boldsymbol{i}$ and $\boldsymbol{i i}$, respectively. $\boldsymbol{B}$, Averaged CS bouton numbers significantly deceased in the ES group and significantly increased after DRX (one-way ANOVA with Dunnett's post hoc test, ${ }^{*} p<0.05$ between ES and control, ${ }^{* *} p<0.01$ between DRX and control; $n=4$ for each group). $C$, Cumulative distribution curve of $C S$ bouton densities of individual interneurons in each group. Inset shows neuronal numbers that are used for analysis in Figure $5, B$ and $C$, which did not show significant difference between groups. $\boldsymbol{D}$, Representative images showing C bouton labeling around motoneurons in each groups. Note much more $C$ bouton contacting motoneuron soma and dendrites in DRX group (Diii) compared with that of control (Di). $\boldsymbol{E}$, C bouton density analysis showed reciprocal change in response to PA manipulation (paired $t$ test between contralateral and ipsilateral sides, $p>0.05$ for control, ${ }^{*} p<0.05$ for ES, ${ }^{* *} p<0.01$ for DRX; $n=4$ for control, $n=7$ for DRX, and $n=5$ for ES). F, Ipsilateral/contralateral ratio of C bouton density in the DRX and ES groups. Scale bars: $A \boldsymbol{i}, 5 \mu \mathrm{m}$; all others, $20 \mu \mathrm{m}$.

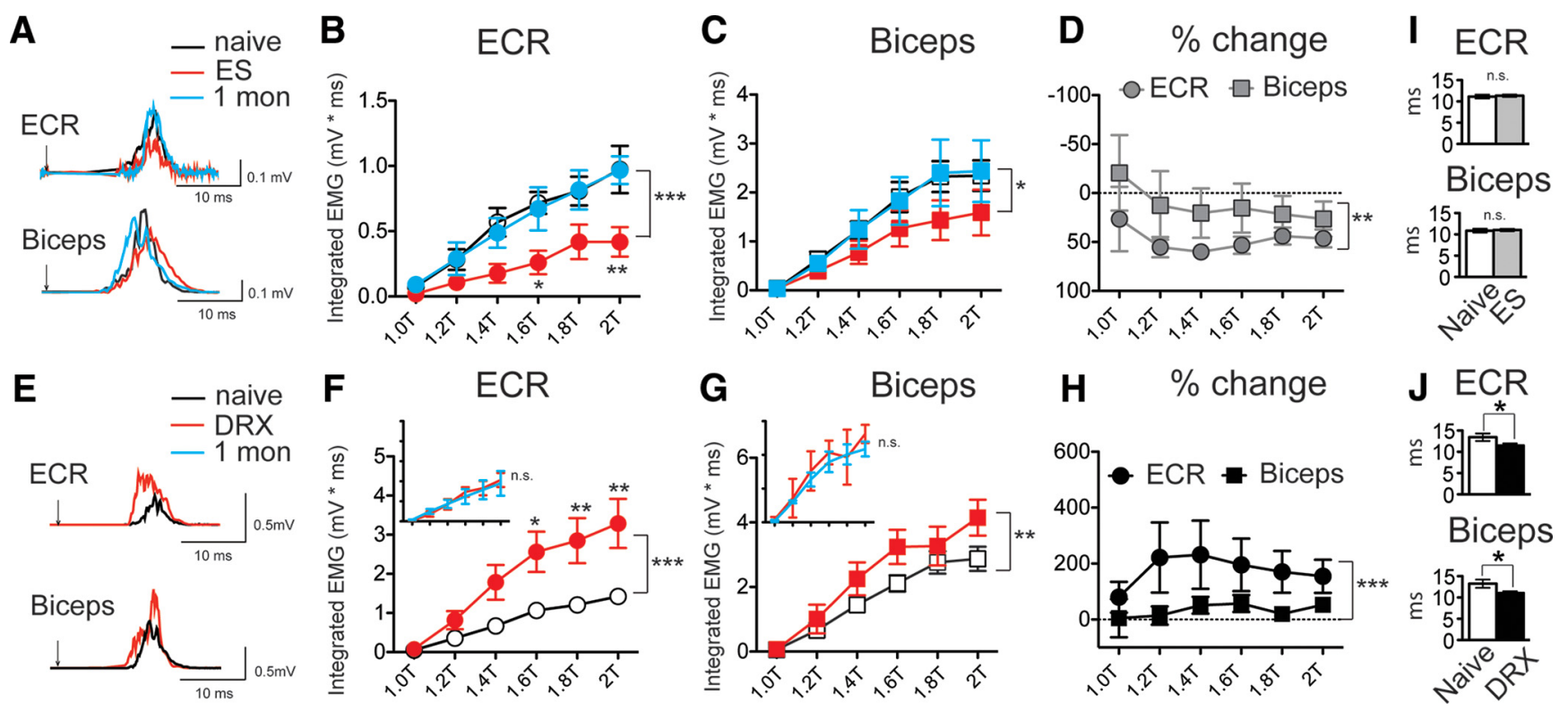

Figure 6. M1 stimulation evoked muscle responses (MEPS) showed functional changes after PA manipulation. $A, E$, Superimposed representative ECR or biceps EMG traces before, right after, and 1 month after $10 \mathrm{~d}$ of ES $(\boldsymbol{A})$, before and 2 weeks after DRX $(\boldsymbol{E})$ in response to 1.4 times of threshold M1 stimulation. All are averaged rectified EMG responses from individual animals in each group. $\boldsymbol{B}, \boldsymbol{C}, \boldsymbol{F}$, and $\boldsymbol{G}$ are EMG recruitment curves from $E S(\boldsymbol{B}, \boldsymbol{C})$ and DRX $(\boldsymbol{F}, \boldsymbol{G})$ animals. $\boldsymbol{B}$ and $\boldsymbol{F}$ are recording from ECR, and $\boldsymbol{C}$ and $\boldsymbol{G}$ are from biceps (two-way ANOVA with Bonferroni's post hoc tests; ES group: $n=4$ and for $\mathrm{ECR},{ }^{* * *} p<0.001$ between three curves; for biceps, ${ }^{* * *} p<0.05$ between three curves; DRX group: $n=6$, and for $\mathrm{ECR},{ }^{* * *} p<0.001$ between two curves, ${ }^{*} p<0.05$ at 1.6 times, ${ }^{* *} p<0.01$ at 1.8 and 2 times; for biceps, ${ }^{* *} p<0.01$ between two curves, no significant difference at individual intensity). Insets in $\boldsymbol{F}$ and $G$ were recordings at 2 weeks and 1 month after DRX from 3 rats. The $x$ - and $y$-axes are the same as in the main recruitment figures; there is no significant difference between 2 weeks and 1 month recordings for either ECR or biceps. $\boldsymbol{D}$ and $\boldsymbol{H}$ compared changes of ECR and biceps responses in ES (D) and 2 weeks DRX $(\boldsymbol{H})$ groups, respectively. Tw0-way ANOVA, ${ }^{* *} p<0.01$ in ES, ${ }^{* * *} p<0.001$ in DRX. I, J, Averaged onset latency of EMG responses in ES $(\boldsymbol{I})$ and DRX $(J)$ animals in response to 1.4 times threshold stimulation. In the ES group, naive ECR: mean of $11.13 \pm 0.4576$ and ES ECR: mean of $11.33 \pm 0.2684$; naive biceps: mean of $10.87 \pm 0.4485$ and ES biceps: mean of $11.02 \pm 0.2812$, paired $t$ test, $p>0.05$. In the DRX group, naive ECR: mean of $13.22 \pm 0.8061$ and DRX ECR: mean of $11.26 \pm 0.3520$; naive biceps: mean of $12.68 \pm 0.7086$; DRX biceps: mean of $10.79 \pm 0.3354$, paired $t$ test, ${ }^{*} p<0.05$. 
In contrast, DRX animals showed much larger MEP amplitudes in both ECR and biceps 2 weeks after DRX (Fig. 6E-G). Three rats were followed until 1 month after DRX, and we found that MEP amplitude in both muscles remained at the higher level as recorded at 2 weeks after DRX (Fig. 6E, F, insets). This suggests that CST sprouting and increased connection strength is persistent, at least for as long as afferents are lost. In addition, ECR muscle showed a significantly greater increase (up to a doubling) compared with that of biceps (up to $\sim 50 \%$ increase; Fig. $6 H$ ). This points to a higher susceptibility to sensory deprivation in spinal motor circuit that control distal forelimb muscles.

DRX leads to an earlier onset of EMG responses in both ECR and biceps. Threepulse epidural stimulation (at 1.4 times threshold) showed an $\sim 2$ ms reduction in latency compared with the naive condition (Fig. $6 E, J$ ). We also tested the latency change with single-pulse epidural stimulation in another set of three animals to limit the effect of temporal facilitation in recruiting larger responses (Fig. 7). Single-pulse epidural motor cortex stimulation is similar to stimulus-triggered averaging using microstimulation (Liang et al., 1993; Park et al., 2001). We took advantage of the implanted epidural M1 electrode to perform analogous single-pulse stimulation. Based on a total of $\sim 450$ EMG responses evoked from three animals, we found that the latency distribution shifted to significantly shorter values in the DRX group (Fig. 7). EMG responses with latency as short as $8 \mathrm{~ms}$ were detected in the DRX group (Fig. 7A). Intriguingly, the findings using both three pulses and single-pulse stimulation are consistent with the observed direct cortico-motoneuronal synapses after DRX. In ES animals, onset latencies remained unchanged for both muscles (Fig. 6I). The reciprocal changes in MEP responses with DRX and ES show that the loss of sensory afferents strengthened connections between the motor cortex and motoneurons, whereas promoting proprioceptive inputs weakens the connection.

\section{Discussion}

We established two experimental competitions between primary afferent fibers and CST axons. In one, increasing primary afferent fiber activity by chronic ES produces afferent fiber sprouting and mostly eliminated CST connection through axonal withdrawal, the winner and loser of the competition, respectively. In the other, eliminating primary afferent fibers by DRX produced more CST connection into the affected spinal cord. Although DRX is a one-sided competition, the observed increase in CST connection mirrors the decrease to expanded afferent fiber connections with ES. The spinal circuit elements examined after DRX - CST functional connections, CST contacts on cholinergic interneurons, and the density of C boutons on motoneurons-all changed in the opposite direction to what is observed with ES. We propose that competition between the CST and primary afferent inputs directs the maintenance of their mature spinal sensorymotor circuits. Knowledge of this competition informs the process of CST axon sprouting and withdrawal after injury. Competition may thus be harnessed to promote motor systems repair.

\section{B Frequency distribution}

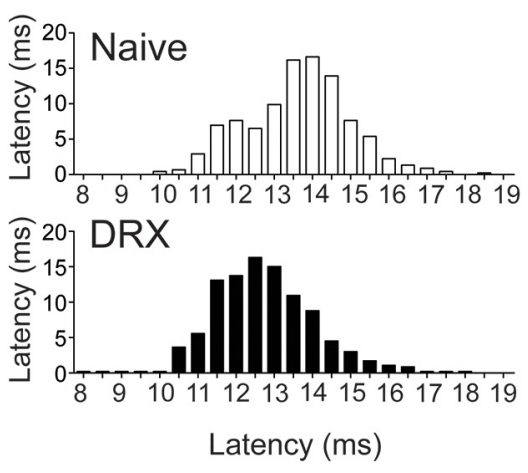

Latency (ms)

Latency (ms)

Figure 7. Single-pulse epidural stimulation reveals shortened cortico-motoneuronal connection after DRX. A, Scattered latency distribution was plotted from a total of $\sim 450$ MEP recordings in ECR muscles from three animals before and after DRX. Black lines are mean values for each group, which is significant shorter after DRX (unpaired $t$ test, ${ }^{* * *} p<0.001$ ). Note there are recordings with latency of $<10 \mathrm{~ms}$. $\boldsymbol{B}$, Frequency distribution of MEP latency of ECR after DRX left-shifted compared with that of naive.

\section{Competition from sensory afferents produces CS axon sprouting and reorganization of the corticospinal motor circuit}

ES is selective for the afferents innervating the traced muscle and is well suited to probing competitive interactions. We showed that PAs from the stimulated muscle direct activity-dependent withdrawal of CST axons. ES produced a significant expansion of ECR afferent fiber projections in the dorsal horn and a doubling of contacts on motoneurons. This, in turn, was accompanied by a substantial loss of CST projections and an $\sim 50 \%$ reduction in muscle connection strength. Expansion of the afferent fiber gray matter distribution in the dorsal horn helps explain the concomitant loss of CST dorsal horn projections with ES, because both share topographic space and even converge on the same interneurons (Jankowska, 1992). The combination of substantial axon loss without local presynaptic density loss explains the large reduction in CST connection strength. Activating mechanosensory afferents does not induce microglia proliferation in the spinal cord as reported previously (Hathway et al., 2009). Thus, we think that non-neural factors are not the major player in CST withdrawal. Rather, the activity-dependent expansion of afferent fiber projections is able to outcompete the CST projections.

As for the complementary experiments, pharmacological approaches have been used to block muscle nerve conduction to reduce activity-dependent effects, but this is often associated with axon damage (Cangiano and Lutzemberger, 1980). We decided to use DRX because it is a robust intervention. We show that DRX increased CST presynaptic sites and nearly doubled functional motor output. This increase is consistent with previous findings showing CST sprouting after partial digit deafferentation (Darian-Smith et al., 2013) but not completely parallel because that study showed axon length increases; in our study, DRX did not result in a greater CST dorsal horn axon length. Sensory denervation also induces plastic changes in the motor cortex. Restricting forelimb movements in monkeys (Milliken et al., 2013) shifted the motor representation from disused to used muscles. Cat studies using distal cutaneous denervation also showed increased MEPs in unaffected proximal muscles, suggesting increased corticospinal efficacy (Bretzner and Drew, 2005). In that study, cortical and spinal plasticity were both shown to contribute to increased corticospinal efficacy to unaffected proximal muscles. In contrast, in our study, sensory denervation elevated motor thresholds and reduced the distal forelimb representation 
(Jiang et al., 2013), suggesting that the increased motor output after DRX is predominantly attributable to strengthened spinal cord CST connection.

\section{CST connections with ChAT interneurons and $\mathrm{C}$ boutons are sensitive to afferent fiber manipulations}

$\mathrm{ChAT}^{+}$spinal interneurons include a distinctive class based on Pitx $2^{+}$gene expression during development (Zagoraiou et al., 2009). Although these cells cannot be identified definitively in the rat, their neurotransmitter phenotype and location suggest that at least some of these interneurons are of the Pitx2 group. Pitx2 ChAT interneurons directly innervate motoneurons, forming $\mathrm{C}$ boutons (Nagy et al., 1993). C boutons are associated with $\mathrm{M}_{2}$ muscarinic activation, which leads to a persistent inward current, thereby increasing the gain of the motoneuron membrane to synaptic inputs (Hellstrom et al., 2003). ES reorganized CSTspinal motor circuits by reducing the density of CST projections onto both motoneurons and $\mathrm{ChAT}^{+}$interneurons and producing a $15 \%$ decrease in the number of $\mathrm{C}$ boutons on motoneurons. This change, coupled with a global retraction of CS axons, could lead to a substantial decrease in the ability of the CST to produce muscle contraction. DRX induced an opposite change in CS projection to $\mathrm{ChAT}^{+}$interneurons and $\mathrm{C}$ boutons on motoneurons; this could increase CST capacity to produce muscle contraction.

Interestingly, development of $\mathrm{ChAT}^{+}$interneurons shows an activity-dependent CST regulation (Chakrabarty et al., 2009). We hypothesize that CS axons exert a trophic modulation of cholinergic interneurons in maturity. In our study, both CST bouton numbers on cholinergic interneurons and $\mathrm{C}$ boutons on motoneurons were modulated in opposite directions by DRX and ES, paralleling the directions of global CS bouton number changes these manipulations produced. Thus, we propose that the reconstruction of CST terminals consequently modulate cholinergic interneurons attributable to trophic effects. Because $\sim 5 \%$ of motoneuron soma membrane is occupied by la afferent terminals (Ichiyama et al., 2006), freeing up this space after DRX could contribute directly to this $\mathrm{C}$ bouton increase. Changes in $\mathrm{C}$ bouton density would not only affect the capacity of the CST to activate muscle but also that of other descending pathways and spinal interneurons because cholinergic interneurons have a variety of input sources and output targets (Zagoraiou et al., 2009), Moreover, many other interneurons in spinal motor circuits receive direct CS and/or afferent fiber innervation, such as Ia inhibitory interneurons in proprioceptive sensorimotor circuits (Hultborn and Santini, 1972) and recently defined dI3 excitatory interneurons in cutaneous sensorimotor circuits (Bui et al., 2013). Competition from afferent fibers would thus not only drive dynamic reconstruction of CST axons but also induce rewiring of spinal motor circuits, which could affect motor function.

\section{Both activity imbalance and freeing up synaptic space are important for competition-dependent CST axonal plasticity}

Activity is critical for axonal growth or retraction during development of both the motor (Martin, 2005) and the sensory (Espinosa and Stryker, 2012) systems. The present study demonstrates activity-dependent axonal outgrowth of spinal projections of primary afferent fibers by tonically elevating activity in maturity. CST axon retraction, in contrast, appears to be attributable to a relatively lower-level activity. Furthermore, we show that the changes provoked by ES are fully reversible physiologically once ES is stopped and animals are followed for 1 month. Plausibly, the activity-dependent afferent fiber outgrowth is subsequently withdrawn and the activity-dependent CST withdrawal is fol- lowed by outgrowth, similar to what we observed after DRX. If so, this outgrowth would also be driven by the relative increase in activity, not an absolute activity increase because there is no exogenous activation of the CST. This is the first demonstration of cyclical regression and strengthening of motor connections driven by targeted activation of a competing pathway in maturity. Just as in development, these reciprocal changes are dependent on an activity imbalance.

The availability of synaptic space is apt to be an important factor in enabling activity-dependent changes. Deafferentation studies show significant mechanosensory terminal elimination $7 \mathrm{~d}$ after injury (Oliveira et al., 2003; Alvarez et al., 2004). We also found loss of PAs 2 weeks after DRX. As afferent axons degenerate, synaptic space is progressively freed, offering the opportunity for CST axonal outgrowth and formation of new connections. Motoneurons, for example, have $\sim 25 \%$ of their surface soma membrane without synaptic boutons (Ichiyama et al., 2006). Activity-dependent presynaptic outgrowth and synapse formation may take advantage of this free space. After injury, such as PTX (Brus-Ramer et al., 2007) or DRX, more free space becomes available. Large-scale deafferentation after DRX drives the original CST projection to form $80 \%$ more synaptic boutons in the gray matter. For ES, in which presumably there is no release of synaptic space, there is only $40 \%$ more of afferent fiber boutons on motoneurons. Combined freeing synaptic space and activation may yield greater CST outgrowth than either alone. The physiological and molecular mechanisms contributing to spaceactivity interactions in axon sprouting and withdrawal remain to be determined in future experiments.

Competitive interactions seem not limited to between PA and CS axons. Injury to peripheral nerves induces hyperexcitability and subsequent sprouting of spared mechanosensory afferents to the denervated superficial spinal dorsal horn (Woolf et al., 1992; Sun et al., 2005). Hyperactivity and increased availability of synaptic space likely contribute to this competitive sprouting. A developmental study showed that weakening of PA inputs on Renshaw cells is accompanied by strengthening of motor neuronal inputs. Proliferation of cholinergic inputs was considered to be responsible for PA weakening, which likely reflects a competition-dependent developmental mechanism (Mentis et al., 2006). It remains to be determined whether a competitiondependent rewiring of Renshaw cell inputs persists in maturity.

Competitive interactions-both activity and space dependent-appear to be an essential driving force for CST remodeling after somatic afferent manipulations and for reorganization of spinal motor circuits and motor function. Our findings provide insight into the reciprocal changes induced by unilateral PTX on spared CST axons and have implications for therapy after spinal injury. Afferent fiber stimulation, which occurs as part of functional ES therapy and passive range of motion approaches (Doucet et al., 2012), may set up a competition between afferent fibers and the CST. If the afferent fibers win, it may help promote the excitability of spinal circuits after injury but at the expense of the CST and other motor pathways. Understanding the logic of competition-dependent spinal plasticity will help predict pathway changes after injury and help inspire new ways to modify the strength of connections of particular pathways to promote rehabilitation after brain or spinal cord injury.

\section{References}

Alstermark B, Ogawa J, Isa T (2004) Lack of monosynaptic corticomotoneuronal EPSPs in rats: disynaptic EPSPs mediated via reticulospinal neurons and polysynaptic EPSPs via segmental interneurons. J Neurophysiol 91:1832-1839. CrossRef Medline 
Alvarez FJ, Villalba RM, Zerda R, Schneider SP (2004) Vesicular glutamate transporters in the spinal cord, with special reference to sensory primary afferent synapses. J Comp Neurol 472:257-280. CrossRef Medline

Arber S, Ladle DR, Lin JH, Frank E, Jessell TM (2000) ETS gene Er81 controls the formation of functional connections between group Ia sensory afferents and motor neurons. Cell 101:485-498. CrossRef Medline

Asante CO, Martin JH (2013) Differential joint-specific corticospinal tract projections within the cervical enlargement. PLoS One 8:e74454. CrossRef Medline

Barber RP, Phelps PE, Houser CR, Crawford GD, Salvaterra PM, Vaughn JE (1984) The morphology and distribution of neurons containing choline acetyltransferase in the adult rat spinal cord: an immunocytochemical study. J Comp Neurol 229:329-346. CrossRef Medline

Blesch A, Tuszynski MH (2002) Spontaneous and neurotrophin-induced axonal plasticity after spinal cord injury. Prog Brain Res 137:415-423. CrossRef Medline

Bretzner F, Drew T (2005) Changes in corticospinal efficacy contribute to the locomotor plasticity observed after unilateral cutaneous denervation of the hindpaw in the cat. J Neurophysiol 94:2911-2927. CrossRef Medline

Brus-Ramer M, Carmel JB, Chakrabarty S, Martin JH (2007) Electrical stimulation of spared corticospinal axons augments connections with ipsilateral spinal motor circuits after injury. J Neurosci 27:13793-13801. CrossRef Medline

Bui TV, Akay T, Loubani O, Hnasko TS, Jessell TM, Brownstone RM (2013) Circuits for grasping: spinal dI3 interneurons mediate cutaneous control of motor behavior. Neuron 78:191-204. CrossRef Medline

Cangiano A, Lutzemberger L (1980) Partial denervation in inactive muscle effects innervated and denervated fibres equally. Nature 285:233-235. CrossRef Medline

Carmel JB, Berrol LJ, Brus-Ramer M, Martin JH (2010) Chronic electrical stimulation of the intact corticospinal system after unilateral injury restores skilled locomotor control and promotes spinal axon outgrowth. J Neurosci 30:10918-10926. CrossRef Medline

Cellerino A, Siciliano R, Domenici L, Maffei L (1992) Parvalbumin immunoreactivity: a reliable marker for the effects of monocular deprivation in the rat visual cortex. Neuroscience 51:749-753. CrossRef Medline

Cesa R, Strata P (2009) Axonal competition in the synaptic wiring of the cerebellar cortex during development and in the mature cerebellum. Neuroscience 162:624-632. CrossRef Medline

Chakrabarty S, Shulman B, Martin JH (2009) Activity-dependent codevelopment of the corticospinal system and target interneurons in the cervical spinal cord. J Neurosci 29:8816-8827. CrossRef Medline

Curfs MH, Gribnau AA, Dederen PJ (1994) Selective elimination of transient corticospinal projections in the rat cervical spinal cord gray matter. Brain Res Dev Brain Res 78:182-190. CrossRef Medline

Darian-Smith C, Lilak A, Alarcón C (2013) Corticospinal sprouting occurs selectively following dorsal rhizotomy in the macaque monkey. J Comp Neurol 521:2359-2372. CrossRef Medline

Deppmann CD, Mihalas S, Sharma N, Lonze BE, Niebur E, Ginty DD (2008) A model for neuronal competition during development. Science 320: 369-373. CrossRef Medline

Donatelle JM (1977) Growth of the corticospinal tract and the development of placing reactions in the postnatal rat. J Comp Neurol 175:207-231. CrossRef Medline

Doucet BM, Lam A, Griffin L (2012) Neuromuscular electrical stimulation for skeletal muscle function. Yale J Biol Med 85:201-215. Medline

Drew T, Prentice S, Schepens B (2004) Cortical and brainstem control of locomotion. Prog Brain Res 143:251-261. CrossRef Medline

Du Beau A, Shakya Shrestha S, Bannatyne BA, Jalicy SM, Linnen S, Maxwell DJ (2012) Neurotransmitter phenotypes of descending systems in the rat lumbar spinal cord. Neuroscience 227:67-79. CrossRef Medline

Espinosa JS, Stryker MP (2012) Development and plasticity of the primary visual cortex. Neuron 75:230-249. CrossRef Medline

Friel KM, Martin JH (2005) Role of sensory-motor cortex activity in postnatal development of corticospinal axon terminals in the cat. J Comp Neurol 485:43-56. CrossRef Medline

Friel K, Chakrabarty S, Kuo HC, Martin J (2012) Using motor behavior during an early critical period to restore skilled limb movement after damage to the corticospinal system during development. J Neurosci 32: 9265-9276. CrossRef Medline

Gibson CL, Clowry GJ (1999) Retraction of muscle afferents from the rat ventral horn during development. Neuroreport 10:231-235. CrossRef Medline

Hathway GJ, Vega-Avelaira D, Moss A, Ingram R, Fitzgerald M (2009) Brief, low frequency stimulation of rat peripheral C-fibres evokes prolonged microglial-induced central sensitization in adults but not in neonates. Pain 144:110-118. CrossRef Medline

Hellström J, Oliveira AL, Meister B, Cullheim S (2003) Large cholinergic nerve terminals on subsets of motoneurons and their relation to muscarinic receptor type 2. J Comp Neurol 460:476-486. CrossRef Medline

Hultborn H, Santini M (1972) Supraspinal control of monosynaptically activated group Ia interneurones in the ventral horn. Acta Physiol Scand 84:142-144. CrossRef Medline

Ichiyama RM, Broman J, Edgerton VR, Havton LA (2006) Ultrastructural synaptic features differ between alpha- and gamma-motoneurons innervating the tibialis anterior muscle in the rat. J Comp Neurol 499:306-315. CrossRef Medline

Jankowska E (1992) Interneuronal relay in spinal pathways from proprioceptors. Prog Neurobiol 38:335-378. CrossRef Medline

Jiang YQ, Williams PT, Martin JH (2013) Rapid and persistent impairments of the forelimb motor representations following cervical deafferentation in rats. Eur J Neurosci 38:3702-3711. CrossRef Medline

Liang F, Rouiller EM, Wiesendanger M (1993) Modulation of sustained electromyographic activity by single intracortical microstimuli: comparison of two forelimb motor cortical areas of the rat. Somatosens Mot Res 10:51-61. CrossRef Medline

Li Q, Martin JH (2000) Postnatal development of differential projections from the caudal and rostral motor cortex subregions. Exp Brain Res 134: 187-198. CrossRef Medline

Li Q, Martin JH (2002) Postnatal development of connectional specificity of corticospinal terminals in the cat. J Comp Neurol 447:57-71. CrossRef Medline

Marsden CD, Rothwell JC, Day BL (1984) The use of peripheral feedback in the control of movement. Trends Neurosci 7:253-257. CrossRef

Martin JH (2005) The corticospinal system: from development to motor control. Neuroscientist 11:161-173. CrossRef Medline

Martin JH, Lee SJ (1999) Activity-dependent competition between developing corticospinal terminations. Neuroreport 10:2277-2282. CrossRef Medline

Martin JH, Kably B, Hacking A (1999) Activity-dependent development of cortical axon terminations in the spinal cord and brain stem. Exp Brain Res 125:184-199. CrossRef Medline

Mentis GZ, Siembab VC, Zerda R, O’Donovan MJ, Alvarez FJ (2006) Primary afferent synapses on developing and adult Renshaw cells. J Neurosci 26:13297-13310. CrossRef Medline

Miles GB, Hartley R, Todd AJ, Brownstone RM (2007) Spinal cholinergic interneurons regulate the excitability of motoneurons during locomotion. Proc Natl Acad Sci U S A 104:2448-2453. CrossRef Medline

Milliken GW, Plautz EJ, Nudo RJ (2013) Distal forelimb representations in primary motor cortex are redistributed after forelimb restriction: a longitudinal study in adult squirrel monkeys. J Neurophysiol 109: 1268-1282. CrossRef Medline

Nagy JI, Yamamoto T, Jordan LM (1993) Evidence for the cholinergic nature of C-terminals associated with subsurface cisterns in alphamotoneurons of rat. Synapse 15:17-32. CrossRef Medline

Niu J, Ding L, Li JJ, Kim H, Liu J, Li H, Moberly A, Badea TC, Duncan ID, Son YJ, Scherer SS, Luo W (2013) Modality-based organization of ascending somatosensory axons in the direct dorsal column pathway. J Neurosci 33:17691-17709. CrossRef Medline

Oliveira AL, Hydling F, Olsson E, Shi T, Edwards RH, Fujiyama F, Kaneko T, Hökfelt T, Cullheim S, Meister B (2003) Cellular localization of three vesicular glutamate transporter mRNAs and proteins in rat spinal cord and dorsal root ganglia. Synapse 50:117-129. CrossRef Medline

Oudega M, Perez MA (2012) Corticospinal reorganization after spinal cord injury. J Physiol 590:3647-3663. CrossRef Medline

Park MC, Belhaj-Saiff A, Gordon M, Cheney PD (2001) Consistent features in the forelimb representation of primary motor cortex in rhesus macaques. J Neurosci 21:2784-2792. Medline

Personius KE, Balice-Gordon RJ (2002) Activity-dependent synaptic plas- 
ticity: insights from neuromuscular junctions. Neuroscientist 8:414-422. CrossRef Medline

Sakatani K, Iizuka H, Young W (1990) Somatosensory evoked potentials in rat cerebral cortex before and after middle cerebral artery occlusion. Stroke 21:124-132. CrossRef Medline

Saling M, Sitárová T, Vejsada R, Hník P (1992) Reaching behavior in the rat: absence of forelimb peripheral input. Physiol Behav 51:1151-1156. CrossRef Medline

Silver J, Schwab ME, Popovich PG (2015) Central nervous system regenerative failure: role of oligodendrocytes, astrocytes, and microglia. Cold Spring Harb Perspect Biol 7:a020602. CrossRef Medline

Song W, Truong DQ, Bikson M, Martin JH (2015) Transspinal direct current stimulation immediately modifies motor cortex sensorimotor maps. J Neurophysiol 113:2801-2811. CrossRef Medline

Stepien AE, Tripodi M, Arber S (2010) Monosynaptic rabies virus reveals premotor network organization and synaptic specificity of cholinergic partition cells. Neuron 68:456-472. CrossRef Medline

Sun Q, Tu H, Xing GG, Han JS, Wan Y (2005) Ectopic discharges from injured nerve fibers are highly correlated with tactile allodynia only in early, but not late, stage in rats with spinal nerve ligation. Exp Neurol 191:128-136. CrossRef Medline

Tan AM, Chakrabarty S, Kimura H, Martin JH (2012) Selective corticospinal tract injury in the rat induces primary afferent fiber sprouting in the spinal cord and hyperreflexia. J Neurosci 32:12896-12908. CrossRef Medline

Valtschanoff JG, Weinberg RJ, Rustioni A (1993) Amino acid immunoreactivity in corticospinal terminals. Exp Brain Res 93:95-103. Medline

Woolf CJ, Shortland P, Coggeshall RE (1992) Peripheral nerve injury triggers central sprouting of myelinated afferents. Nature 355:75-78. CrossRef Medline

Yang HW, Lemon RN (2003) An electron microscopic examination of the corticospinal projection to the cervical spinal cord in the rat: lack of evidence for cortico-motoneuronal synapses. Exp Brain Res 149: 458-469. Medline

Zagoraiou L, Akay T, Martin JF, Brownstone RM, Jessell TM, Miles GB (2009) A cluster of cholinergic premotor interneurons modulates mouse locomotor activity. Neuron 64:645-662. CrossRef Medline 